\title{
VERIFICACIÓN DE LAS PREDICCIONES DEL MODELO MOCAGE UTILIZANDO EL ÍNDICE EUROPEO DE CALIDAD DEL AIRE
}

Coralina Hernández Trujillo(1), Alberto Cansado(1), Isabel Martínez ${ }^{(1)}$.

${ }^{(1)}$ Agencia Estatal de Meteorología (AEMET), Área de aplicaciones, chernandezt@aemet.es

\section{Introducción}

La transmisión de información al público sobre el estado de la contaminación del aire ha recibido una creciente atención en los planes de calidad del aire, ya que una de las tareas que debe llevarse a cabo por parte de las administraciones para cumplir con las directivas europeas es poner a disposición del público la información adecuada sobre la calidad del aire.

En este aspecto, la Agencia Estatal de Meteorología proporciona información sobre la composición química de la atmósfera mediante un sistema de predicción de la calidad del aire con el que se realizan predicciones operativas de diferentes contaminantes sobre la península ibérica y Baleares. Dicho sistema de predicción se basa en el modelo de transporte químico MOCAGE (Modèle de Chimie Atmosphérique de Grande Échelle), desarrollado por Météo-France y utilizado en AEMET en virtud de un acuerdo de colaboración con el citado servicio meteorológico. Actualmente, el sistema utiliza el inventario de emisiones TNO-MACCIII (2011), que es la referencia más actual en inventarios de alta resolución sobre Europa que hay disponible.

Las predicciones se elaboran diariamente y comprenden diferentes especies químicas como son el ozono $\left(\mathrm{O}_{3}\right)$, el dióxido de nitrógeno $\left(\mathrm{NO}_{2}\right)$, el monóxido de nitrógeno $(\mathrm{NO})$, el monóxido de carbono $(\mathrm{CO})$ o el dióxido de azufre $\left(\mathrm{SO}_{2}\right)$. Recientemente además, se han incluido dos nuevas variables de material particulado de tamaños menores de 10 micras y menores de 2,5 micras, el PM10 y el PM2.5 respectivamente, tras implantar una nueva versión del modelo que incluye el pronóstico de aerosoles.

Sin embargo, esta información puede llegar a ser demasiado técnica y compleja si tenemos en cuenta que debe informar a un público que no tiene por qué ser experto en materia de calidad del aire. Es por ello que el siguiente objetivo es presentar las predicciones de calidad del aire de una manera más clara, sencilla y comprensible a través de un índice de calidad del aire.

Un índice de calidad del aire es un valor adimensional, establecido a partir de la información procedente de las directivas vigentes relacionadas con los distintos contaminantes atmosféricos. En nuestro caso, el objetivo es presentar un índice diario de calidad del aire calculado con las predicciones que se obtienen de manera operativa mediante el sistema de predicción de calidad del aire de AEMET.

Se han utilizado diferentes índices como el Air Quality Index for Health o AQIH (http://www.epa.ie/air/quality/index/) de la Agencia Medioambiental de Irlanda (EPA) y el índice europeo de calidad del aire, desarrollado por la Agencia Europea de Medioambiente en base a las directivas europeas vigentes sobre calidad del aire (http://www. eea.europa.eu/themes/air/air-quality-index/index). 
El modelo MOCAGE proporciona valores previstos de concentración de las especies químicas citadas anteriormente. Con ellos se calcula un índice parcial para cada especie considerada en el índice europeo, que son el ozono $\left(\mathrm{O}_{3}\right)$, el dióxido de nitrógeno $\left(\mathrm{NO}_{2}\right)$, el dióxido de azufre $\left(\mathrm{SO}_{2}\right)$ y el material particulado PM10 y PM2.5. Para generar el índice global, que integra la información de todos los contaminantes, se selecciona el valor máximo de los índices parciales en cada punto.

En este trabajo analizaremos si el índice europeo de calidad del aire previsto a partir de las predicciones del modelo se ajusta o no a la realidad observada. Para ello, contamos con las observaciones de las redes de calidad del aire gestionadas por las comunidades autónomas y entidades locales, que obtenemos a través del MAPAMA (Ministerio de Agricultura y Pesca, Alimentación y Medio Ambiente). Se ha realizado una verificación objetiva comparando el índice que se obtiene a partir de las predicciones en cada una de las estaciones de observación con el índice calculado a partir de los datos de observación de cada estación.

\section{Sistema MOCAGE-AEMET}

El modelo MOCAGE (Modèle de Chimie Atmosphérique de Grande Échelle) es un modelo global que proporciona simulaciones numéricas de los procesos dinámicos, físicos y químicos en la troposfera y baja estratosfera. Tiene una estructura vertical de 47 niveles híbridos que se extienden desde la superficie hasta $0.1 \mathrm{hPa}$ (aproximadamente $60 \mathrm{~km}$ ) y la resolución horizontal del modelo parte de un dominio global a $2^{\circ} \times 2^{\circ} \mathrm{con}$ capacidad para trabajar con hasta 3 dominios anidados adicionales.

El esquema químico usado es el denominado RACMOBUS que comprende 119 especies y 372 reacciones fotoquímicas. Este esquema es una combinación del esquema de RACM para la química troposférica (Stockwell et al., 1997) y del esquema de química estratosférica REPROBUS (Lefêvre et al., 1994).

La configuración operativa del modelo en AEMET consta de un dominio global a $2^{\circ}$ de resolución horizontal sobre el que se anida un dominio europeo a $0,5^{\circ}$ y finalmente un dominio peninsular a $0,1^{\circ}$.
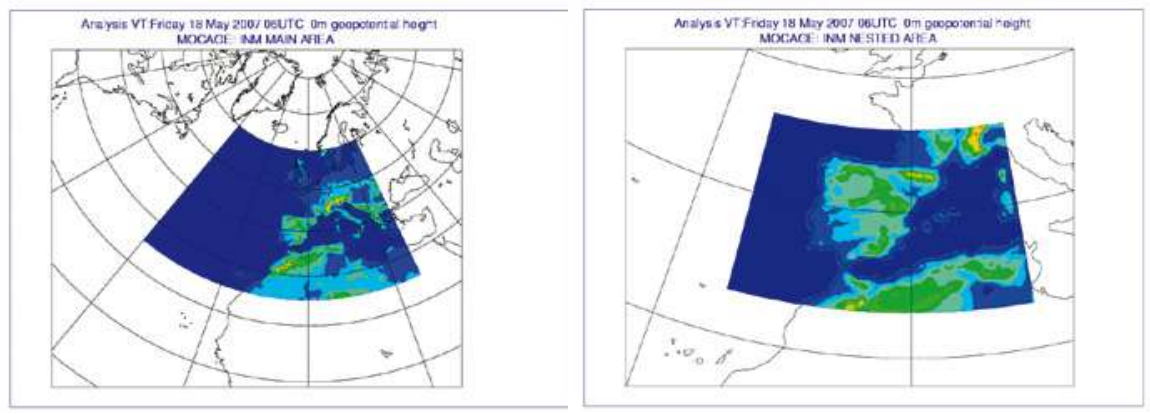

Fig. 1.- Dominios europeo $\left(0,5^{\circ}\right)$ y peninsular $\left(0,1^{\circ}\right)$ de la configuración operativa del sistema MOCAGE-AEMET. 
El sistema MOCAGE-AEMET es un modelo offline que precisa datos meteorológicos que proceden de diversos análisis y predicciones de los modelos numéricos meteorológicos. Los forzamientos meteorológicos globales provienen del modelo IFS del Centro Europeo de Predicción a Plazo Medio (ECMWF) y de ARPEGE de Météo-France. Los forzamientos para los dominios de $0,5^{\circ}$ y $0,1^{\circ}$ provienen del modelo HIRLAM HNR de AEMET de $0,05^{\circ}$ para el dominio peninsular $\left(0.1^{\circ}\right)$ y de HIRLAM ONR de AEMET de $0,16^{\circ}$ para el dominio europeo $\left(0,5^{\circ}\right)$.

En lo referido a las emisiones, MOCAGE puede utilizar diferentes inventarios de emisiones dependiendo del dominio espacial (global, regional, alta resolución) en el que nos encontremos. Las emisiones son un punto clave en los resultados obtenidos por los modelos de transporte químico, por lo que deben ser elegidos de forma cuidadosa y estar lo más actualizados posible (Arrizabalaga et al., 2014). Actualmente, el sistema MOCAGE-AEMET utiliza el inventario de emisiones generado por la empresa holandesa TNO para el proyecto MACCIII, que toma como año base el 2011, siendo la referencia más actual en inventarios de alta resolución sobre Europa que hay disponible.

El modelo se ejecuta operativamente en AEMET dos veces al día con un alcance de predicción de 24 horas. Las predicciones comprenden diferentes especies químicas como son el ozono $\left(\mathrm{O}_{3}\right)$, el dióxido de nitrógeno $\left(\mathrm{NO}_{2}\right)$, el monóxido de nitrógeno $(\mathrm{NO})$, el monóxido de carbono $(\mathrm{CO})$ o el dióxido de azufre $\left(\mathrm{SO}_{2}\right)$. Recientemente además, se han incluido dos nuevas variables de material particulado de tamaños menores de 10 micras y menores de 2,5 micras, el PM10 y el PM2.5 respectivamente, tras implantar una nueva versión del modelo que incluye el pronóstico de aerosoles.

Los resultados de las predicciones en superficie para las especies citadas anteriormente son publicados en la página web de AEMET (http://www.aemet.es/es/eltiempo/ prediccion/calidad_del_aire). Las concentraciones aparecen expresadas en $\mu \mathrm{g} / \mathrm{m}^{3}$, salvo el contenido total de la columna de $\mathrm{O}_{3}$ que aparece expresado en Unidades Dobson.

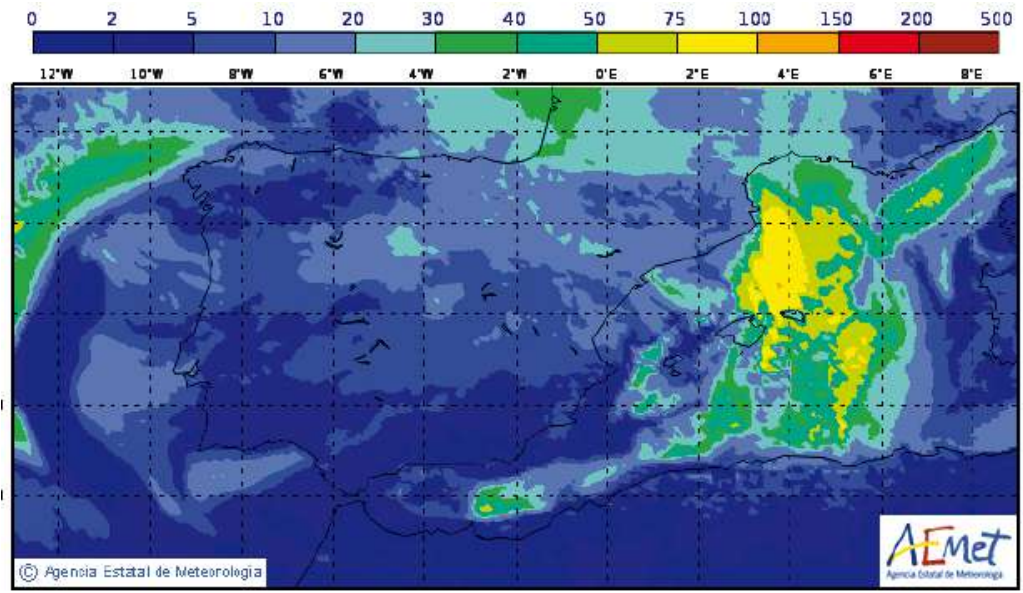

Fig. 2.-

Concentración prevista de PM10 en superficie para una hora determinada, expresada en $\mu \mathrm{g} / \mathrm{m}_{3}$. 


\section{3. Índices de calidad del aire}

Un índice de calidad del aire es un valor adimensional, establecido a partir de la información procedente de las directivas vigentes relacionadas con los distintos contaminantes atmosféricos con el que se pretende conseguir hacer un resumen sobre el estado de la calidad del aire, facilitando al público la comprensión e interpretación de los valores cuantitativos de los diferentes contaminantes atmosféricos.

A cada valor de concentración (observado o previsto) de un contaminante se le asocia otro valor adimensional, conocido como índice, perteneciente a una escala. Además, cada valor de esta escala lleva asociado una etiqueta descriptiva ("buena", "regular", "mala", etc.) y un color (rojo si la calidad es mala, verde si es buena, etc.), lo que facilita su interpretación cuando se representa en un mapa.

Los distintos índices existentes tienen en cuenta diferentes contaminantes atmosféricos aunque los más comunes son: el ozono $\left(\mathrm{O}_{3}\right)$, el dióxido de nitrógeno $\left(\mathrm{NO}_{2}\right)$, el dióxido de azufre $\left(\mathrm{SO}_{2}\right)$ y el material particulado PM10 y PM2.5.

También presentan diferentes rangos, así por ejemplo el Air Quality Index for Health o AQIH (http://www.epa.ie/air/quality/index/) de la Agencia Medioambiental de Irlanda (EPA) presenta una escala de 10 niveles (Tabla 2) y el índice europeo de calidad del aire (Tabla 1), desarrollado por la Agencia Europea de Medioambiente en base a las directivas europeas vigentes sobre calidad del aire (http://www.eea.europa.eu/themes/ air/air-quality-index/index), presenta tan solo 5 niveles.

\begin{tabular}{|l|cccccc|} 
& $\begin{array}{c}\text { Indice Europeo } \\
\text { medias }\left(\underline{\mu g} / \mathrm{m}^{3}\right)\end{array}$ & $\begin{array}{c}\mathbf{O}_{3} \\
\text { móvil } 8 \mathrm{~h}\end{array}$ & $\begin{array}{c}\mathrm{NO}_{2} \\
\mathrm{hh}^{2}\end{array}$ & $\begin{array}{c}\mathrm{SO}_{2} \\
\mathrm{hh}^{2}\end{array}$ & $\begin{array}{c}\text { PM10 } \\
\text { móvil 24h }\end{array}$ & $\begin{array}{c}\text { PM2.5 } \\
\text { móvil 24h }\end{array}$ \\
\hline Muy Buena & 1 & $0-80$ & $0-40$ & $0-100$ & $0-20$ & $0-10$ \\
\hline Buena & 2 & $80-120$ & $40-100$ & $100-200$ & $20-35$ & $10-20$ \\
\hline Moderada & 3 & $120-180$ & $100-200$ & $200-350$ & $35-50$ & $20-25$ \\
\hline Mala & 4 & $180-240$ & $200-400$ & $350-500$ & $50-100$ & $25-50$ \\
\hline Muy Mala & 5 & $>240$ & $>400$ & $>500$ & $>100$ & $>50$ \\
\hline
\end{tabular}

Tabla 1.- Esquema del índice europeo desarrollado por la Agencia Europea de Medioambiente.

\begin{tabular}{|c|c|c|c|c|c|c|}
\hline & $\begin{array}{c}\text { Indice AQIH } \\
\text { medias }(\mu \mathrm{g} / \mathrm{m} 3)\end{array}$ & $\begin{array}{c}0_{3} \\
\text { móvil 8h }\end{array}$ & $\begin{array}{l}\mathrm{NO}_{2} \\
1 \mathrm{~h}\end{array}$ & $\begin{array}{l}\mathrm{SO}_{2} \\
\mathrm{hh}^{2}\end{array}$ & $\begin{array}{c}\text { PM10 } \\
\text { móvil 24h }\end{array}$ & $\begin{array}{c}\text { PM2.5 } \\
\text { móvil 24h }\end{array}$ \\
\hline \multirow{3}{*}{ Buena } & 1 & 0-33 & $0-67$ & $0-29$ & 0-16 & $0-11$ \\
\hline & 2 & $33-66$ & $67-134$ & $29-59$ & $16-33$ & $11-23$ \\
\hline & 3 & $66-100$ & $134-200$ & $59-89$ & $33-50$ & $23-35$ \\
\hline \multirow{3}{*}{ Aceptable } & 4 & $100-120$ & $200-267$ & $89-119$ & $50-58$ & $35-41$ \\
\hline & 5 & $120-140$ & $267-334$ & $119-149$ & $58-66$ & $41-47$ \\
\hline & 6 & $140-160$ & $334-400$ & $149-179$ & $66-75$ & $47-53$ \\
\hline \multirow{3}{*}{ Mala } & 7 & $160-187$ & $400-467$ & $179-256$ & $75-83$ & $53-58$ \\
\hline & 8 & $187-213$ & $467-554$ & 256-295 & $83-91$ & $58-64$ \\
\hline & 9 & $213-240$ & $554-600$ & 295-334 & $91-100$ & $64-70$ \\
\hline Muy Mala & 10 & $>240$ & $>600$ & $>334$ & $>100$ & $>70$ \\
\hline
\end{tabular}

Tabla 2.- Esquema del Air Quality Index for Health de la Agencia Medioambiental de Irlanda. 
Actualmente no existe un índice único a nivel nacional que sea utilizado por las distintas administraciones responsables, aunque una de las medidas previstas en el Plan Nacional de Calidad del Aire 2017-2019 (Plan Aire II) es precisamente la adopción de un índice de calidad del aire a nivel nacional, homogéneo y comprensible.

Esta medida es muy importante ya que el uso de diferentes índices de calidad del aire, con distintos intervalos o categorías y con códigos de colores distintos puede crear confusión entre la población, al hacer que la información sea dispersa y no comparable en términos cualitativos. En la figura 3, podemos observar las diferencias de los mapas de los valores previstos por el sistema MOCAGE-AEMET para el índice europeo y para el AQIH para un mismo día.
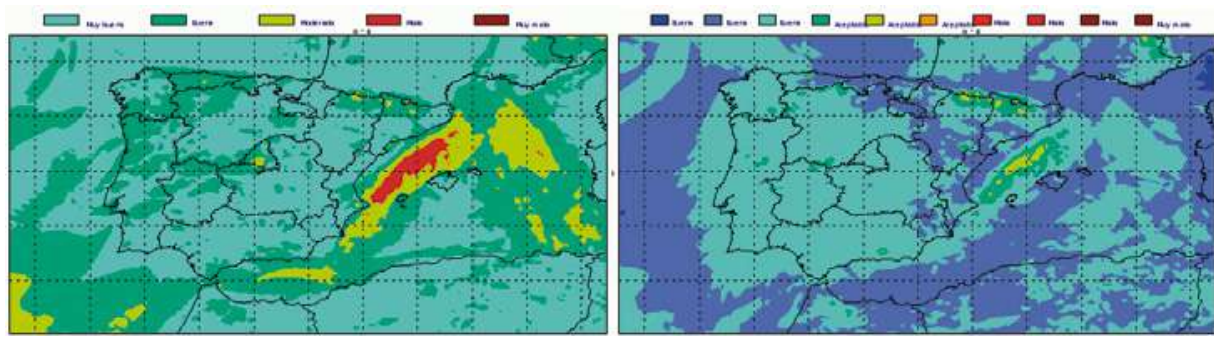

Fig. 3.- Mapa del índice de calidad europeo (izq.) y mapa del AQIH (dcha.) previsto por el sistema MOCAGE-AEMET para el 19/12/2017.

El cálculo del índice a través de los valores previstos de concentración de las diferentes especies químicas por el modelo MOCAGE requiere, en primer lugar, calcular un índice parcial para cada especie considerada en el índice que se vaya a utilizar. En este caso, ambos índices tienen en cuenta los mismos contaminantes: el ozono $\left(\mathrm{O}_{3}\right)$, el dióxido de nitrógeno $\left(\mathrm{NO}_{2}\right)$, el dióxido de azufre $\left(\mathrm{SO}_{2}\right)$ y el material particulado PM10 y PM2.5.

Cada especie requiere un tratamiento diferenciado. Por ejemplo, el índice para el $\mathrm{NO}_{2}$ y el $\mathrm{SO}_{2}$ viene determinado por el valor máximo entre los 24 valores horarios disponibles para un día. Para el ozono se calculan las medias móviles octohorarias para cada hora del día y el valor máximo de éstas es el que determina su índice diario. Por último, para el caso del material particulado, para cada hora del día considerado se calculan las medias móviles de las últimas 24 horas y el índice se define en función del valor máximo de dichas medias móviles.

Finalmente, para generar el índice global, que integra la información de todos los contaminantes, se selecciona el valor máximo de los índices parciales en cada punto.

\section{Verificación del Índice Europeo de Calidad del Aire}

Para evaluar si el índice europeo de calidad del aire realizado con las predicciones del modelo se ajusta o no a la realidad observada, se ha realizado una verificación objetiva comparando el índice que se obtiene a partir de las predicciones en cada una de las estaciones de observación con el índice calculado a partir de los datos de observación de cada estación. 
Dichas observaciones proceden de las redes de calidad del aire gestionadas por las comunidades autónomas y entidades locales y las obtenemos a través del MAPAMA (Ministerio de Agricultura y Pesca, Alimentación y Medio Ambiente).

Los resultados de la verificación que se presenta están basados en el cálculo de medidas de exactitud y skill scores para variables multi-categóricas que han surgido como generalización a los ya conocidos para variables categóricas (Joliffe and Stephenson, 2012). Todas las medidas calculadas se obtienen a través de tablas de contingencia que resumen el número de predicciones y observaciones en las diferentes categorías (Muy Buena, Buena, Moderada, Mala y Muy Mala).

A continuación presentamos las tablas de contingencia que se han obtenido para cada especie $\left(\mathrm{NO}_{2}, \mathrm{SO}_{2}, \mathrm{O}_{3}, \mathrm{PM} 10\right.$ y PM2.5) en los períodos de tiempo comprendidos entre el 21/06/2017 y el 22/09/2017 (verano), y el 22/09/2017 y el 21/12/2017 (otoño) usando el índice europeo de calidad del aire (Tabla 1). Se han utilizado la versión operativa del modelo para realizar la verificación del $\mathrm{NO}_{2}, \mathrm{SO}_{2}, \mathrm{O}_{3}$, mientras que para el PM10 y el PM2.5 se han usado las predicciones de la pasada experimental con aerosoles que incluye polvo desértico, que a partir del mes de noviembre coincide con la pasada operativa del modelo.

\subsection{Resultados de la verificación para el $\mathrm{NO}_{2}$}

En los datos correspondientes al primer periodo verificado (Tabla 3) podemos observar que prácticamente el 91,47\% de ellos se encuentran en las categorías Muy Buena y Buena, donde el $67,44 \%$ se corresponde con predicciones correctas. En las categorías Moderada, Mala y Muy Mala se cometen muy pocos errores en este período, siendo el más alto un 3,74\% de predicciones en la categoría Moderada correspondientes a observaciones en la categoría Buena.

Con respecto al segundo período verificado (Tabla 4), destaca la disminución de datos en las dos primeras categorías, pues baja a $82,64 \%$ del total. Disminuye notablemente el porcentaje de aciertos en la primera categoría (de un $55,22 \%$ baja a un $34,55 \%$ ), pero aumentan los aciertos en la categoría Buena (un 5,54\%).

\begin{tabular}{|l|cccccc|}
\hline Observaciones & Muy Buena & Buena & Moderada & Mala & Muy Mala & Total Predicciones \\
\hline Muy Buena & 55,22 & 15,62 & 0,73 & 0,02 & 0,01 & 71,6 \\
\hline Buena & 8,41 & 12,22 & 1 & 0,01 & 0 & 21,64 \\
\hline Moderada & 1,17 & 3,74 & 1,17 & 0,02 & 0 & 6,09 \\
\hline Mala & 0,05 & 0,3 & 0,29 & 0,01 & 0 & 0,65 \\
\hline Muy Mala & 0 & 0,01 & 0,01 & 0 & 0 & 0,02 \\
\hline Total Observaciones & 64,85 & 31,88 & 3,2 & 0,06 & 0,01 & 100 \\
\hline
\end{tabular}

Tabla 3.- Tabla de contingencia en porcentajes para el $\mathrm{NO}_{2}$ correspondiente al período 21/06/201722/09/2017. 
También aumentan los errores en las categorías Moderada, Mala y Muy Mala, destacando sobre todo los errores que se comenten al predecir las categorías Muy Buena y Buena cuando se observa la categoría Moderada (2,42\% y 3,57\% de los datos, respectivamente) y el aumento del error al predecir la categoría Moderada cuando se observa la categoría Buena $(4,42 \%)$.

\begin{tabular}{|l|cccccc|}
\hline Observaciones & Muy Buena & Buena & Moderada & Mala & Muy Mala & Total Predicciones \\
\hline Muy Buena & 34,55 & 23,88 & 2,42 & 0,03 & 0 & 60,88 \\
\hline Buena & 6,45 & 17,76 & 3,57 & 0,04 & 0 & 27,82 \\
\hline Moderada & 0,84 & 4,42 & 2,91 & 0,13 & 0 & 8,3 \\
\hline Mala & 0,04 & 0,55 & 1,54 & 0,19 & 0 & 2,32 \\
\hline Muy Mala & 0 & 0,01 & 0,05 & 0,17 & 0 & 0,68 \\
\hline Total Observaciones & 41,88 & 46,62 & 10,94 & 0,56 & 0 & 100 \\
\hline
\end{tabular}

Tabla 4.- Tabla de contingencia en porcentajes para el $\mathrm{NO}_{2}$ correspondiente al período 22/09/2017$21 / 12 / 2017$.
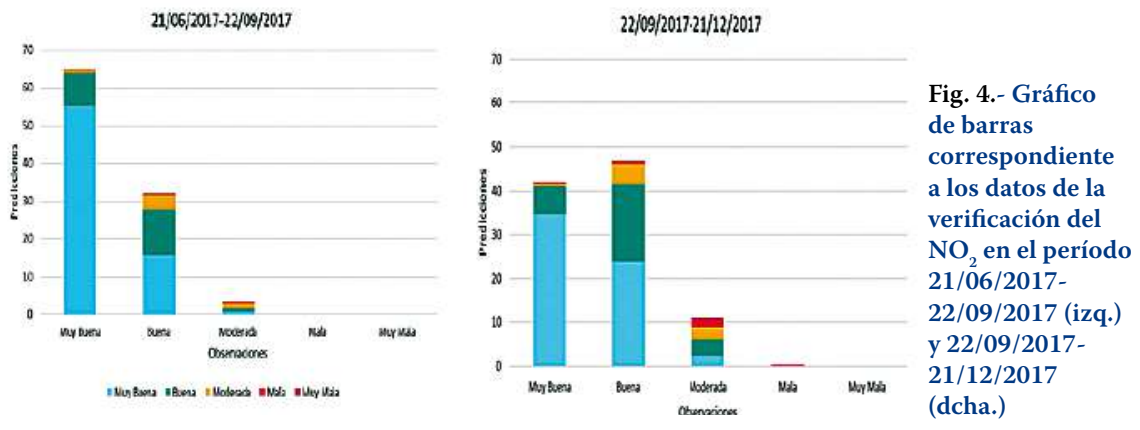

\subsection{Resultados de la verificación para el $\mathrm{SO}_{2}$}

Los resultados de la verificación del índice para el $\mathrm{SO}_{2}$ (Tabla 5 y Tabla 6) son bastante similares en los dos períodos estudiados. En general, se obtiene un porcentaje muy alto de aciertos en la primera categoría del índice $(96,99 \%$ y $97,18 \%$ en el primer y segundo período de tiempo verificado, respectivamente) y muy pocos errores, de los que los más destacables son los cometidos al predecir la categoría Buena cuando se observa Muy Buena (2,16\% y $1,92 \%)$.

El hecho de que en los últimos años se hayan reducido considerablemente los niveles de $\mathrm{SO}_{2}$ en España y que, además, en el índice europeo de calidad del aire (Tabla 1) concentraciones de hasta $100 \mu \mathrm{g} / \mathrm{m}^{3}$ de $\mathrm{SO}_{2}$ entren dentro de la categoría Muy Buena, explica el comportamiento de los datos de la verificación del índice previsto para este contaminante. 


\begin{tabular}{|l|cccccc|}
\hline Observaciones & Muy Buena & Buena & Moderada & Mala & Muy Mala & Total Predicciones \\
\hline Predicciones & & & & & & 97,58 \\
\hline Buy Buena & 96,99 & 0,49 & 0,09 & 0,01 & 0 & 2,19 \\
\hline Moderada & 2,16 & 0,02 & 0,01 & 0 & 0 & 0,23 \\
\hline Mala & 0,21 & 0,02 & 0 & 0 & 0 & 0 \\
\hline Muy Mala & 0 & 0 & 0 & 0 & 0 & 0 \\
\hline Total Observaciones & 99,36 & 0,53 & 0,1 & 0,01 & 0 & 100 \\
\hline
\end{tabular}

Tabla 5.- Tabla de contingencia en porcentajes para el $\mathrm{SO}_{2}$ correspondiente al período 21/06/201722/09/2017.

\begin{tabular}{|l|cccccc|}
\hline Observaciones & Muy Buena & Buena & Moderada & Mala & Muy Mala & Total Predicciones \\
\hline Muy Buena & 97,18 & 0,41 & 0,1 & 0 & 0,01 & 97,7 \\
\hline Buena & 1,92 & 0,03 & 0 & 0 & 0 & 1,95 \\
\hline Moderada & 0,3 & 0 & 0 & 0 & 0 & 0,3 \\
\hline Mala & 0,05 & 0 & 0 & 0 & 0 & 0,05 \\
\hline Muy Mala & 0 & 0 & 0 & 0 & 0 & 0 \\
\hline Total Observaciones & 99,45 & 0,44 & 0,1 & 0,01 & 0 & 100 \\
\hline
\end{tabular}

Tabla 6.- Tabla de contingencia en porcentajes para el $\mathrm{SO}_{2}$ correspondiente al período 22/09/201721/12/2017.
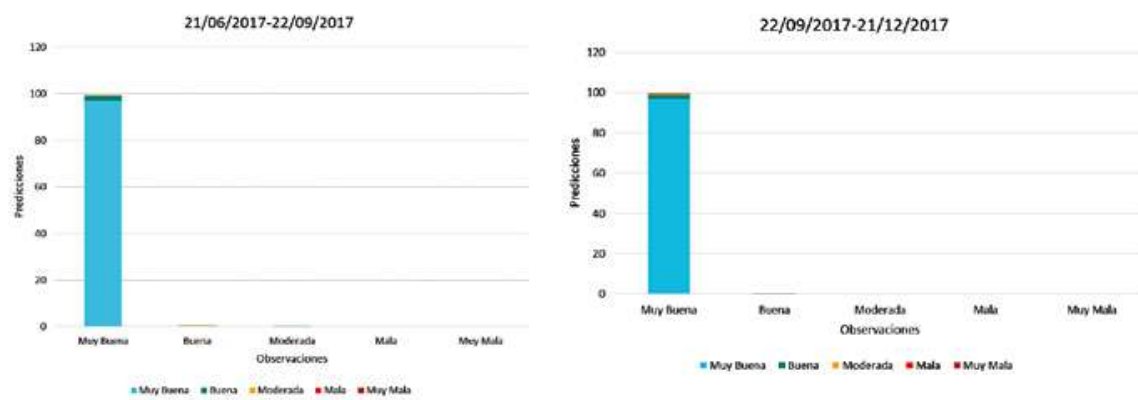

Fig. 5.- Gráfico de barras correspondiente a los datos de la verificación del $\mathrm{SO}_{2}$ en el período 21/06/2017-22/09/2017 (izq.) y 22/09/2017-21/12/2017 (dcha.).

\subsection{Resultados de la verificación para el $\mathrm{O}_{3}$}

En el caso del ozono, podemos observar que en verano (Tabla 7) el 65,73\% de los datos se encuentra en las dos primeras categorías, mientras que en otoño (Tabla 8) este porcentaje aumenta a un $97,87 \%$. Esta mejoría se debe, en gran medida, al aumento de predicciones correctas en la categoría Muy Buena, que pasa de ser un 13,56\% a un $50,34 \%$. 


\begin{tabular}{|l|cccccc|}
\hline Observaciones & Muy Buena & Buena & Moderada & Mala & Muy Mala & Total Predicciones \\
\hline Muy Buena & 13,56 & 13,25 & 1,68 & 0,02 & 0,01 & 28,52 \\
\hline Buena & 10,04 & 28,88 & 8,37 & 0,11 & 0,02 & 47,42 \\
\hline Moderada & 2,84 & 10,79 & 6,24 & 0,08 & 0,01 & 19,96 \\
\hline Mala & 0,29 & 1,16 & 1,58 & 0,04 & 0 & 3,07 \\
\hline Muy Mala & 0,07 & 0,3 & 0,66 & 0 & 0 & 1,03 \\
\hline Total Observaciones & 26,8 & 54,38 & 18,53 & 0,25 & 0,04 & 100 \\
\hline
\end{tabular}

Tabla 7.- Tabla de contingencia en porcentajes para el $\mathrm{O}_{3}$ correspondiente al período 21/06/201722/09/2017.

\begin{tabular}{|c|c|c|c|c|c|c|}
\hline Observaciones & Muy Buena & Buena & Moderada & Mala & Muy Mala & Total Predicciones \\
\hline Muy Buena & 50,34 & 8,38 & 0,1 & 0 & 0,01 & 58,83 \\
\hline Buena & 24,23 & 14,92 & 0,27 & 0 & 0 & 39,42 \\
\hline Moderada & 0,53 & 1,2 & 0,02 & 0 & 0 & 1,75 \\
\hline Mala & 0 & 0 & 0 & 0 & 0 & 0,05 \\
\hline Muy Mala & 0 & 0 & 0 & 0 & 0 & 0 \\
\hline Total Observacione & 75,1 & 24,5 & 0,39 & 0 & 0,01 & 100 \\
\hline
\end{tabular}

Tabla 8.- Tabla de contingencia en porcentajes para el $\mathrm{O}_{3}$ correspondiente al período 22/09/2017$21 / 12 / 2017$.
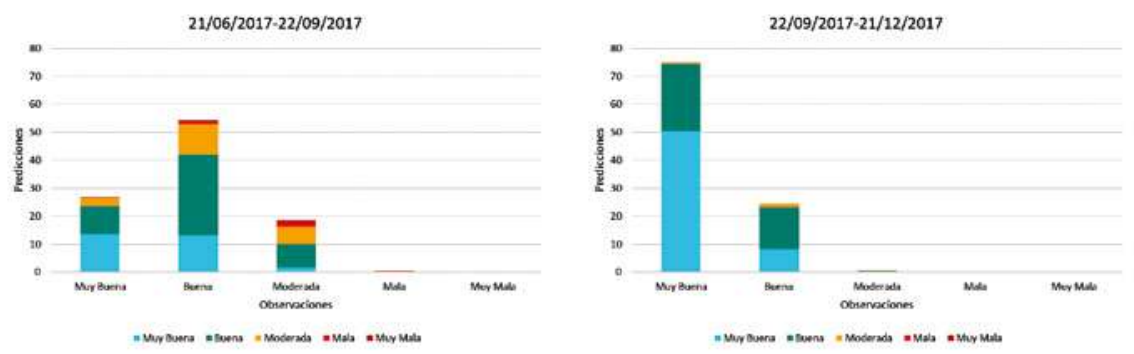

Fig. 6.- Gráfico de barras correspondiente a los datos de la verificación del $\mathrm{O}_{3}$ en el período 21/06/2017-22/09/2017 (izq.) y 22/09/2017-21/12/2017 (dcha.).

En los errores cometidos fuera de las categorías Muy Buena y Buena, destacan los cometidos en verano (Tabla 7) al predecir la categoría Moderada cuando se observa la categoría Muy Buena y Buena (2,84\% y 10,79\%). En otoño, estos errores disminuyen claramente, siendo prácticamente nulos (Tabla 8).

En general, se observa una clara mejoría en los resultados de la predicción del índice para el ozono en el período comprendido entre 22/09/2017 y el 21/12/2017, correspondiente a la estación de otoño. En la verificación correspondiente al verano, vemos que el modelo presenta un comportamiento más irregular, cometiendo más errores que en la estación de otoño. 
El ozono es un contaminante secundario, ya que se forma a partir de ciertos precursores como son los óxidos de nitrógeno $\left(\mathrm{NO}_{\mathrm{x}}\right)$, el monóxido de carbono $(\mathrm{CO})$ o los compuestos orgánicos volátiles (COVs), entre otros. Esta variedad de fuentes y especies precursoras junto con una dinámica compleja (altamente no lineal) hacen que su modelización sea complicada y, por tanto, que los niveles previstos del índice de ozono que se obtienen (sobre todo en verano) no se correspondan en muchos casos con los valores observados del índice.

\subsection{Resultados de la verificación para el PM10}

En las tablas de contingencia para el PM10 vemos que el modelo se comporta de manera similar en ambos períodos verificados. En general, el porcentaje total de predicciones en la categoría Muy Buena es muy alto en las dos estaciones (96,72\% y 95,23\%). Esto muestra que el modelo tiene una tendencia clara a subestimar el índice observado, puesto que los datos observados se concentran, principalmente, en las tres primeras categorías. Este comportamiento del modelo en relación a la subestimación del material particulado es común a la mayoría de los modelos de transporte químico (Konovalov et al., 2009, Stern et al., 2008).

Los errores más grandes aparecen al predecir la categoría Muy Buena cuando se observan la categorías Buena y Moderada (36,5\% y 14,83\%, respectivamente, en verano y $33,77 \%$ y $11,71 \%$, respectivamente, en otoño).

\begin{tabular}{|l|cccccc|}
\hline Observaciones & Muy Buena & Buena & Moderada & Mala & Muy Mala & Total Predicciones \\
\hline Predicciones & & & & & & \\
\hline Muy Buena & 34,94 & 36,5 & 14,83 & 5,05 & 0,4 & 96,72 \\
\hline Buena & 0,71 & 1,03 & 0,69 & 0,52 & 0,03 & 2,98 \\
\hline Moderada & 0,05 & 0,1 & 0,06 & 0,05 & 0,01 & 0,27 \\
\hline Muy Mala & 0 & 0 & 0,01 & 0,01 & 0 & 0,02 \\
\hline Total Observaciones & 0 & 0 & 0,01 & 0 & 0 & 0,01 \\
\hline
\end{tabular}

Tabla 9.- Tabla de contingencia en porcentajes para el PM10 correspondiente al período 21/06/2017-22/09/2017.

\begin{tabular}{|l|cccccc|}
\hline Predicciones & Muy Buena & Buena & Moderada & Mala & Muy Mala & Total Predicciones \\
\cline { 1 - 6 } Muena & 44,43 & 33,77 & 11,71 & 4,88 & 0,44 & 95,23 \\
Buena & 1,21 & 1,64 & 0,89 & 0,57 & 0,03 & 4,34 \\
Moderada & 0,17 & 0,13 & 0,07 & 0,03 & 0,01 & 0,41 \\
Mala & 0,01 & 0,01 & 0 & 0 & 0 & 0,02 \\
Muy Mala & 0 & 0 & 0 & 0 & 0 & 0 \\
\hline Total Observaciones & 45,82 & 35,55 & 12,67 & 5,48 & 0,48 & 100 \\
\hline
\end{tabular}

Tabla 10.- Tabla de contingencia en porcentajes para el PM10 correspondiente al período 22/09/2017-21/12/2017. 

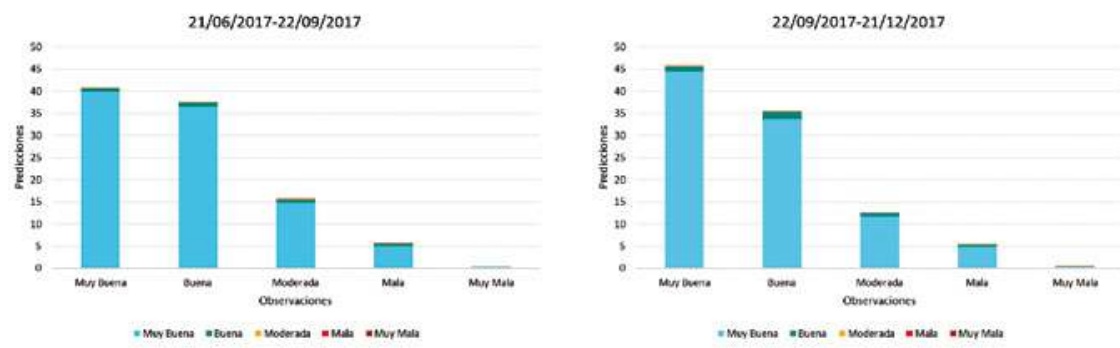

Fig. 7.- Gráfico de barras correspondiente a los datos de la verificación del PM10 en el período 21/06/2017-22/09/2017 (izq.) y 22/09/2017-21/12/2017 (dcha.).

\subsection{Resultados de la verificación para el PM2.5}

En el caso del PM2.5 las tablas de contingencia (Tablas 11 y 12) muestran que el modelo tiene un comportamiento parecido al que hemos visto anteriormente con el PM10. El porcentaje total de predicciones está repartido principalmente entre las categorías Muy Buena y Buena, pero en este caso la mayoría de las observaciones también están repartidas en esas dos categorías, lo que hace que los resultados sean ligeramente mejores que los que obtenemos con el PM10.

\begin{tabular}{|l|cccccc|}
\hline Observaciones & Muy Buena & Buena & Moderada & Mala & Muy Mala & Total Predicciones \\
\hline Predicciones & & & & & & \\
\hline Muy Buena & 49,26 & 29,42 & 2,14 & 1,27 & 0,26 & 82,35 \\
\hline Buena & 5,1 & 9,56 & 1,11 & 1,08 & 0,14 & 16,99 \\
\hline Moderada & 0,19 & 0,35 & 0 & 0 & 0,02 & 0,56 \\
\hline Mala & 0 & 0,06 & 0 & 0 & 0,02 & 0,08 \\
\hline Muy Mala & 0 & 0,02 & 0 & 0 & 0 & 0,02 \\
\hline Total Observaciones & 54,55 & 39,41 & 3,25 & 2,35 & 0,44 & 100 \\
\hline
\end{tabular}

Tabla 11.- Tabla de contingencia en porcentajes para el PM2.5 correspondiente al período 21/06/2017-22/09/2017.

\begin{tabular}{|l|cccccc|}
\hline Predicciones & Muy Buena & Buena & Moderada & Mala & Muy Mala & Total Predicciones \\
\hline Muy Buena & 38,66 & 28,65 & 3,92 & 3,4 & 0,42 & 75,05 \\
\hline Buena & 4,66 & 11,9 & 2,51 & 2,83 & 0,21 & 22,11 \\
\hline Moderada & 0,2 & 0,86 & 0,4 & 0,3 & 0 & 1,76 \\
\hline Mala & 0,02 & 0,48 & 0,35 & 0,23 & 0 & 1,08 \\
\hline Muy Mala & 0 & 0 & 0 & 0 & 0 & 0 \\
\hline Total Observaciones & 43,24 & 41,89 & 7,18 & 6,76 & 0,63 & 100 \\
\hline
\end{tabular}

Tabla 12.- Tabla de contingencia en porcentajes para el PM2.5 correspondiente al período 22/09/2017-21/12/2017. 
En verano se obtienen resultados un poco mejores que en otoño, puesto que se aprecia una disminución del porcentaje de aciertos (pasando de un 58,82\% de aciertos a un 50,96\%) y un aumento de los errores al predecir las categorías Muy Buena y Buena cuando se observa las categorías Moderada, Mala y Muy Mala.
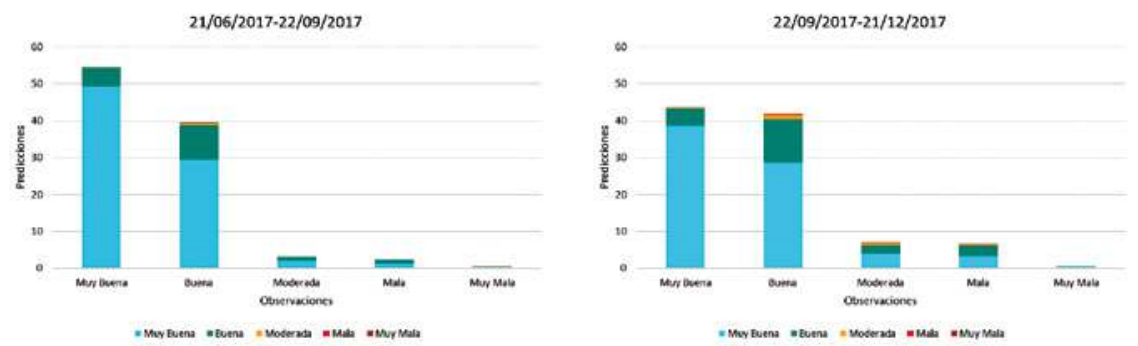

Fig. 8.- Gráfico de barras correspondiente a los datos de la verificación del PM2.5 en el período 21/06/2017-22/09/2017 (izq.) y 22/09/2017-21/12/2017 (dcha.).

\subsection{Medidas de exactitud}

Entre las medidas de exactitud que hemos decidido usar para la verificación del índice se encuentran: la proporción de predicciones correctas (PC) y la probabilidad de detección de cada categoría PODi, donde,

$$
\begin{gathered}
P C=\frac{1}{N} \cdot \sum_{i=1}^{5} n\left(F_{i}, O_{i}\right) \\
P O D_{i}=\frac{n\left(F_{i}, O_{i}\right)}{N\left(O_{i}\right)}, \quad i=1, \ldots, 5 .
\end{gathered}
$$

Siendo $n\left(F_{i}, O_{i}\right)$ el número de predicciones correctas en la categoría i y $N\left(O_{i}\right)$ el número de observaciones en la categoría i.

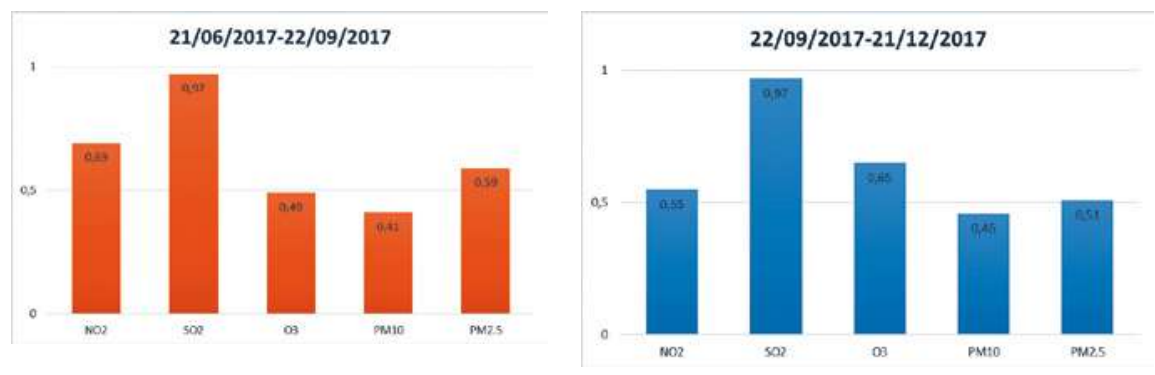

Fig. 9 Proporción de predicciones correctas para cada especie química en el período 21/06/201722/09/2017 (izq.) y 22/09/2017-21/12/2017 (dcha.). 
En la figura 9 podemos observar las proporciones de predicciones correctas en cada estación (verano y otoño) para cada contaminante. Como ya se apreciaba en las tablas de contingencia presentadas anteriormente, la proporción de predicciones correctas es bastante alta en el caso del $\mathrm{SO}_{2}$ en ambas estaciones, mientras que para el PM10 y el PM2.5 se obtienen las proporciones más bajas. En el caso del ozono, podemos ver la mejora de los pronósticos del índice en otoño, ya que la proporción de predicciones correctas pasa de ser 0,49 en verano a un 0,65 en otoño. Con el $\mathrm{NO}_{2}$, ocurre precisamente lo contrario, la proporción es más alta en verano $(0,69)$ que en otoño $(0,55)$.

En los siguientes gráficos de barras (figuras 10 y 11) aparecen representadas las probabilidades de detección de cada categoría para cada contaminante en los períodos verificados. En general, se observa que la categoría Muy Buena tiene una probabilidad alta de detección, sobre todo en el $\mathrm{SO}_{2}$, el PM10 y el PM2.5. En las siguientes categorías, precisamente en estos compuestos, vemos que las probabilidades de detección son muy bajas o nulas, pero se debe a motivos diferentes. En el caso del $\mathrm{SO}_{2}$, lo que ocurre es que ni se observan ni se predicen datos en esas categorías, por lo que no se pueden calcular esas probabilidades. Sin embargo, en el caso del PM10 y el PM2.5 lo que ocurre es que, a pesar de observarse casos en esas categorías, el número de casos pronosticados por el modelo es muy bajo o nulo, por lo que la probabilidad de detección es muy baja o nula en esas categorías.

Por otro lado, el $\mathrm{NO}_{2}$ y el $\mathrm{O}_{3}$, presentan probabilidades más altas de detección en las categorías Buena, Moderada y Mala. La mejora de los pronósticos del índice de $\mathrm{O}_{3}$ en otoño también se aprecia a través de estas probabilidades de detección, ya que aumenta la probabilidad de detectar la categoría Muy Buena, Buena y Moderada y la disminución de la probabilidad de la categoría Mala se debe a que el número de casos observados en dicha categoría es nulo (Tabla 8). Para el $\mathrm{NO}_{2}$, las probabilidades son similares en ambos períodos, destacando el aumento de la probabilidad de detectar la categoría Mala en otoño.

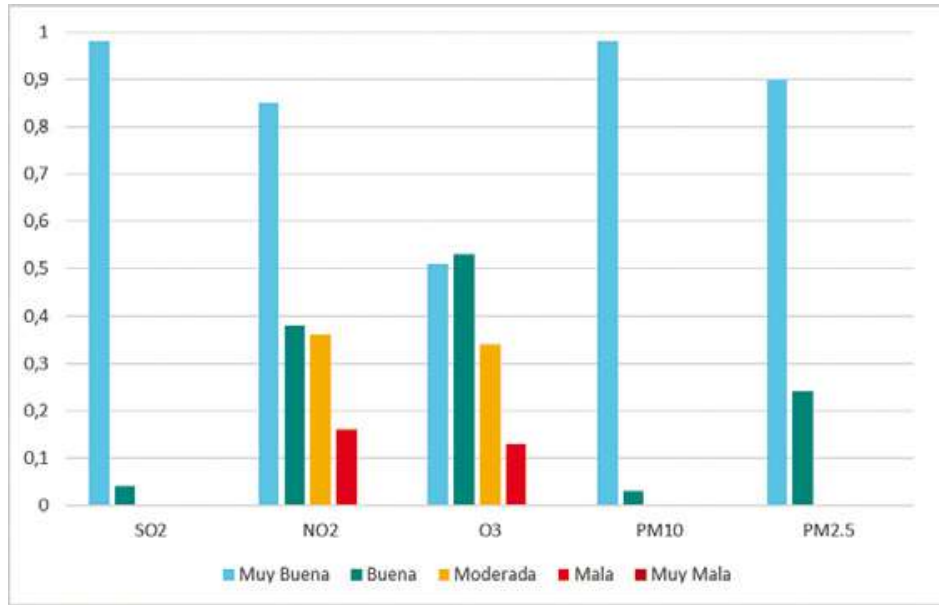

Fig. 10.-

Probabilidad de detección de cada categoría para cada especie química en el período

21/06/201722/09/2017. 


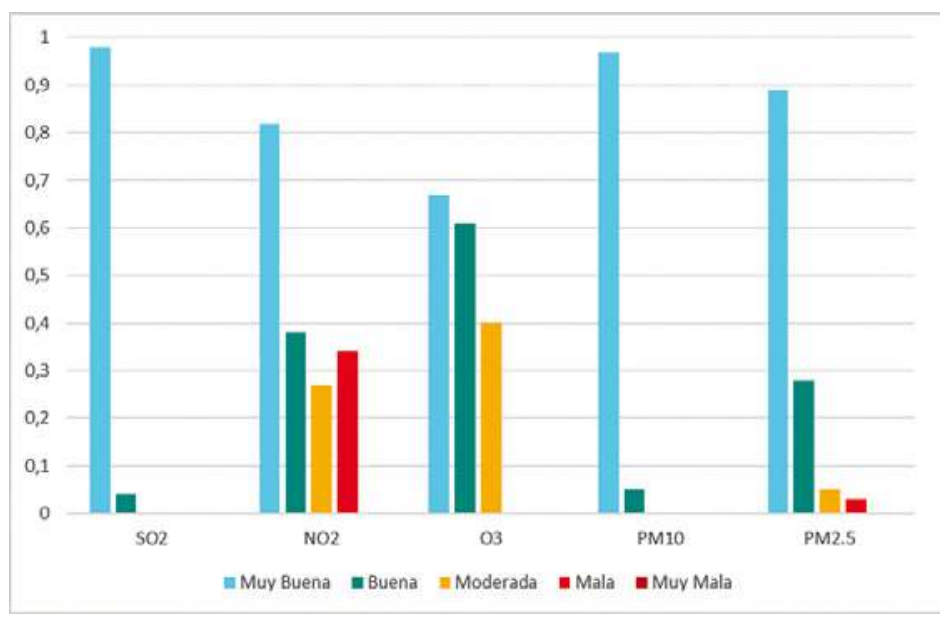

Fig. 11.-

Probabilidad de detección de cada categoría para cada especie química en el período 22/09/201721/12/2017.

\section{Conclusiones}

La implementación de un índice de calidad del aire a las predicciones del sistema MOCAGE-AEMET facilitará en gran medida la interpretación de las previsiones de las concentraciones de los diferentes contaminantes que abarca el modelo, pero los usuarios deben tener claro que los datos que se proporcionan deben ser interpretados como tendencias y en ningún caso son válidos para determinar una posible superación de los valores legislados.

La calidad de las predicciones del índice depende, en general, del contaminante que estemos tratando y, en algunos casos, de la estación del año en la que nos encontremos. Así, la verificación del índice para el $\mathrm{SO}_{2}$ muestra que las predicciones son muy buenas tanto en otoño y en verano, mientras que para el ozono se aprecia una clara variación estacional, subestimando el índice en verano y mejorando las predicciones en otoño. Para el $\mathrm{NO}_{2}$, podemos decir que en ambas estaciones la calidad de las predicciones es aceptable. Por último, en el caso del PM10 y el PM2.5, se observa que se subestima el índice tanto en verano como en otoño, siendo esta subestimación más notable para el PM10.

\section{Agradecimientos}

Por último, queremos agradecer al Ministerio de Agricultura y Pesca, Alimentación y Medio Ambiente habernos permitido acceder a los datos de las estaciones de observación, proporcionados por las diferentes redes nacionales, autonómicas y locales de calidad del aire, para la realización de este trabajo. 


\section{Referencias}

- Arrizabalaga J., Cansado A., Martínez I., 2014: Evaluación del impacto de los inventarios de emisiones en el sistema de predicción de la calidad del aire de AEMET, Congreso Nacional del Medio Ambiente 2014.

- Joliffe, I.T., and Stephenson, D.B., 2012: Forecast Verification: A practicioner's Guide in Atmospheric Science 2nd ed. Wiley-Blackwell, 274 pp.

- Josse, B., Simon, P., and Peuch, V.-H., 2004: Radon global simulations with the multiscale chemistry and transport model MOCAGE. Tellus, 56B, 339-356.

- Konovalov I.B., Beekmann M., Meleux F., Dutot A., Foret G., 2009: Combining deterministic and statistical approaches for PM10 forecasting in Europe, Atmospheric Environment, 43, 6425-6434.

- Kuenen, J. J. P., Visschedijk, A. J. H., Jozwicka, M., and Denier van der Gon, H. A. C., 2014: TNOMACC_II emission inventory; a multi-year (2003-2009) consistent high-resolution European emission inventory for air quality modelling, Atmos. Chem. Phys., 14, 10963-10976, doi:10.5194/ acp-14-10963-2014.

- Lefêvre F., Brasseur G.P., Folkins I., Smith A.K., Simon P., 1994: Chemistry of the 1991-1992 stratospheric winter: three-dimensional model simulations, Journal of Geophysical Research, 99 (D4), 8183-8195.

- Stern R., Builtjes P., Schaap M., Timmermans R., Vautard R., Hodzic A., Memmesheimer M., Feldmann H., Renner E., Wolke R., Kerschbaumer A., 2008: A model inter-comparison study focusing on episodes with elevated PM10 concentrations, Atmospheric Environment, 42, 45674588.

- Stockwell W.R. Kirchner F., Kuhn M., Seefeld S., 1997: A new mechanism for regional atmospheric chemistry modelling, Journal of Geophysical Research, 102, 25847-25879. 